\title{
The posterior parietal cortex and subjectively perceived confidence during memory retrieval
}

\author{
Syanah C. Wynn, ${ }^{1}$ Marc P. H. Hendriks, ${ }^{1,2}$ Sander M. Daselaar, ${ }^{1}$ Roy P. C. Kessels, ${ }^{1}$ \\ and Dennis J. L. G. Schutter ${ }^{1}$ \\ ${ }^{7}$ Donders Institute for Brain, Cognition and Behaviour, Radboud University, 6525 HP Nijmegen, The Netherlands; ${ }^{2}$ Academic Centre \\ of Epileptology, Kempenhaeghe, 5590 AB Heeze, The Netherlands
}

\begin{abstract}
Functional neuroimaging studies suggest a role for the left angular gyrus (AG) in processes related to memory recognition. However, results of neuropsychological and transcranial magnetic stimulation (TMS) studies have been inconclusive regarding the specific contribution of the AG in recollection, familiarity, and the subjective experience of memory. To obtain further insight into this issue, 20 healthy right-handed volunteers performed a memory task in a single-blind withinsubject controlled TMS study. Neuronavigated inhibitory repetitive TMS (rTMS) was applied over the left AG and the vertex in a randomized and counterbalanced order. Prior to rTMS participants were presented with a list of words. After rTMS participants were shown a second list of words and instructed to indicate if the word was already shown prior to rTMS ("old") or was presented for the first time ("new"). In addition, subjectively perceived memory confidence was assessed. Results showed that recollection was unaffected following inhibitory left AG rTMS. In contrast, rTMS over the left AG improved both familiarity and the subjectively perceived confidence of participants that demonstrated low baseline memory recognition. Our study highlights the importance of taking into account individual differences in experimental designs involving noninvasive brain stimulation.
\end{abstract}

Memory recollection includes detailed source information about the remembered event, such as where and when the information was obtained, and is often characterized by a high level of subjectively perceived confidence about its accuracy (Yonelinas and Jacoby 1995; Yonelinas 2002; Woodruff et al. 2006; Mickes et al. 2009). A lower and more variable degree of subjectively perceived confidence in the accuracy of memories occurs in cases of familiarity, when the source information of a memory trace is not available (e.g., Atkinson and Juola 1974; Mandler 1980; Yonelinas and Jacoby 1995; Yonelinas 2002). Behavioral studies indicate that these two distinct retrieval processes in memory recognition can be independently affected by various experimental manipulations (Yonelinas 2001a,b; Yonelinas and Levy 2002; Koen and Yonelinas 2014), suggestive of a categorical distinction between recollection and familiarity.

The distinction between recollection and familiarity has also been demonstrated by functional magnetic resonance imaging (fMRI) studies showing ventrolateral posterior parietal cortex (PPC) activity during recollection and activity of the dorsolateral PPC during familiarity (Ciaramelli et al. 2008; Vilberg and Rugg 2008; Rugg and King 2017). In addition to the unique regional involvement, there is considerable overlap in cortical activity during recollection and familiarity (Skinner and Fernandes 2007; Vilberg and Rugg 2008; Horn et al. 2016; Rugg and King 2017). In particular, the left angular gyrus (AG) of the ventrolateral PPC shows increased activity during both recollection (Skinner and Fernandes 2007; Vilberg and Rugg 2008) and familiarity (Skinner and Fernandes 2007; Horn et al. 2016). Unfortunately however, the correlational nature of functional neuroimaging techniques makes it difficult to make strong inferences about whether or not the left AG is directly implicated in these aspects of memory.

Corresponding author: S.Wynn@donders.ru.nI

Article is online at http://www.learnmem.org/cgi/doi/10.1101/lm.048033.118.
The number of neuropsychological studies that aim to provide a more direct link between the PPC and memory is still limited and has yielded mixed results. While some PPC patients do not show deficits (Ally et al. 2008; Simons et al. 2008), other patients have memory impairments in objective (Ben-Zvi et al. 2015) or subjective recollection (Berryhill et al. 2007; Davidson et al. 2008; Simons et al. 2010; Hower et al. 2014; Ciaramelli et al. 2017). For example, PPC patients with memory impairments have deficits in cued recall (Ben-Zvi et al. 2015), report fewer details of autobiographical memories during free recall (Berryhill et al. 2007; Davidson et al. 2008), and/or make less "remember" responses on a remember/know memory paradigm (Davidson et al. 2008; Ciaramelli et al. 2017). Yet, other studies have found evidence for less confidence in "old" responses (Hower et al. 2014) and reduced source memory confidence (Simons et al. 2010). Heterogeneity of lesions, lack of baseline measurements, and small patient samples make the results sometimes hard to interpret.

In an attempt to address the limitations of functional neuroimaging and patient studies, transcranial magnetic stimulation (TMS) can be used to transiently perturb cortical activity in healthy participants. Subsequently, TMS induced effects on memoryrelated processes can be examined in controlled experimental designs. For instance, facilitatory repetitive TMS (rTMS) over an individualized PPC target can enhance associative memory and spatial recollection (Wang et al. 2014; Nilakantan et al. 2017). In another study, inhibitory continuous theta burst stimulation (cTBS) over the left AG was found to reduce subjectively perceived confidence in source information, while item and source memory performance remained unchanged. This led the authors to suggest that the left AG is involved in the subjective experience of contextual

(C) 2018 Wynn et al. This article is distributed exclusively by Cold Spring Harbor Laboratory Press for the first 12 months after the full-issue publication date (see http://learnmem.cshlp.org/site/misc/terms.xhtml). After 12 months, it is available under a Creative Commons License (Attribution-NonCommercial 4.0 International), as described at http://creativecommons.org/licenses/by-nc/4.0/. 
recollection (Yazar et al. 2014). In a more recent study, cTBS over the left AG did not disrupt item and source memory (Bonni et al. 2015). Unfortunately, subjectively perceived memory confidence was not examined in this study. Another study reported that inhibitory rTMS over the left AG reduced the level of detail of episodic memories and altered the subjective experience of these memories (Thakral et al. 2017a).

While results from neuroimaging studies point to a role for the left AG in recollection and familiarity, lesion and TMS studies hint to a link with the subjective memory experience, such as perceived confidence. To this end, we applied inhibitory rTMS to healthy volunteers in a single-blind crossover design to further explore the role of the left AG in recollection, familiarity, and subjectively perceived memory confidence. Given the role of the left AG in the subjective experience of memories, we hypothesized that inhibitory rTMS over the left AG would lower subjectively perceived memory confidence. Specifically, we expected a lower rate of highconfident responses in the left AG rTMS condition. In addition, based on previous TMS studies (Yazar et al. 2014; Bonni et al. 2015), we did not anticipate a change in familiarity following inhibitory rTMS over the left AG. Finally, a negative effect on recollection in response to the left AG rTMS condition was expected.

\section{Results}

The rTMS procedure was well tolerated by all participants and no adverse effects occurred. At the end of the second session, nine participants indicated that they did not notice any differences between the stimulation of the target (AG) and control (VE) site. The 11 participants that did notice a difference indicated that the stimulation location differed over sessions $(N=2)$, that they had more sensations during VE stimulation $(N=1)$ or left AG stimulation $(N=4)$, or that they performed better after VE stimulation $(N=1)$ or left AG stimulation $(N=3)$. A binomial test showed that the probability of observing 11 out of 20 participants that re-
A
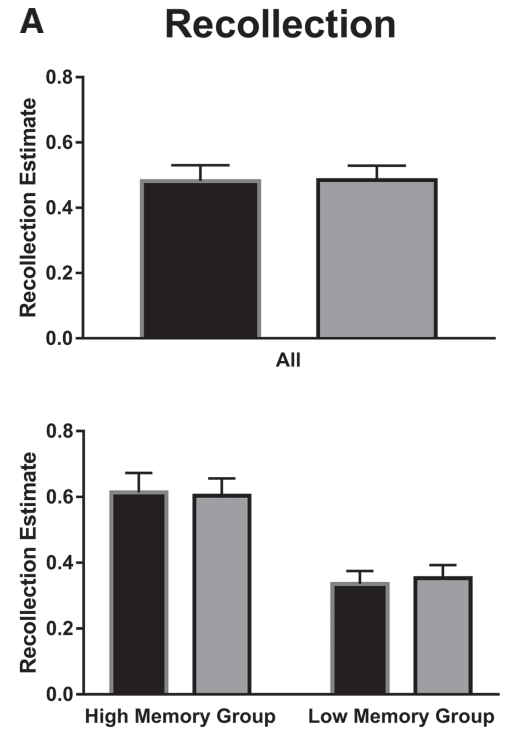

B
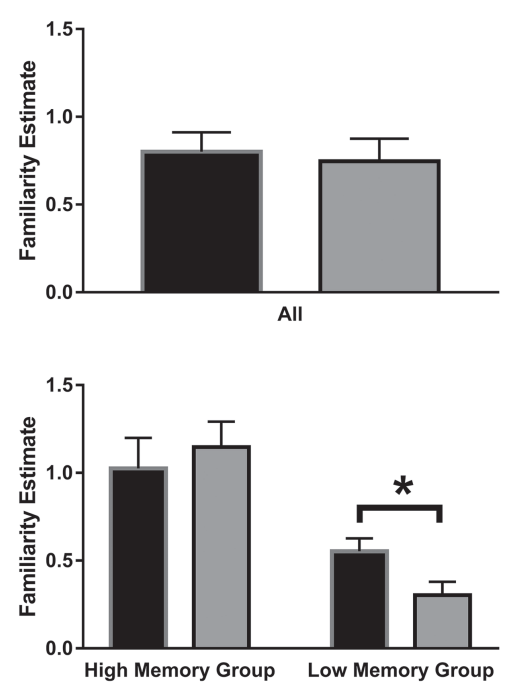

Angular Gyrus $\square$ Vertex

Figure 1. (Top) Mean recollection and familiarity estimates per stimulation condition. (Bottom) Mean recollection and familiarity estimates for low and high performing participants, based on a d-prime median split. Error bars represent standard errors of the mean. A statistical significant difference $(P<$ $0.05)$ is indicated by a star. port a difference between sessions was not significantly different from a $50 \%$ chance level $(P=0.82)$.

\section{Memory recognition}

No significant test-retest effects were found on memory recognition as d-prime $(M=1.37, S D=0.70)$ did not differ significantly between the first and second session $\left(t_{(18)}=0.73, P=0.48\right)$. In addition, d-prime did not differ significantly between angular gyrus and vertex stimulation $\left(\mathrm{t}_{(18)}=0.79, P=0.44\right)$.

\section{Recollection and familiarity}

No main effect of rTMS condition was observed on recollection $\left(F_{(1,18)}=0.10, P=0.92, \eta_{p}{ }^{2}=0.001\right)$ and familiarity $\left(F_{(1,18)}=0.47\right.$, $P=0.50, \eta_{p}{ }^{2}=0.025$, see Fig. 1$)$.

Recent findings suggest that behavioral effects of noninvasive brain stimulation can depend on individual differences in baseline performance (Ridding and Ziemann 2010; Sarkar et al. 2014; Benwell et al. 2015; Klaus and Schutter 2018), therefore we explored in an ad hoc fashion whether baseline memory recognition interacted with the rTMS intervention. To this end we divided the participants into a "low" and a "high" memory recognition group, based on a median split of d-prime scores during VE stimulation. This group categorization was used as a between-subject factor in analyses that explored the interaction between individual differences in memory recognition and the rTMS intervention.

The three-way interaction between stimulation (AG, VE), memory measure (recollection, familiarity), and group (high, low) was significant $\left(F_{(1,17)}=9.62, P=0.006, \eta_{p}^{2}=0.36\right)$. Post-hoc analysis did not reveal an interaction effect between stimulation condition and individual differences on recollection scores $\left(F_{(1,17)}=\right.$ $0.21, P=0.66, \eta_{p}^{2}=0.012$ ). However, a significant interaction effect between rTMS condition and individual differences on familiarity scores was found $\left(F_{(1,17)}=6.98, P=0.017, \eta_{p}{ }^{2}=0.29\right)$. Post-hoc $t$-tests revealed that in the low memory recognition group familiarity was enhanced after left AG rTMS as compared to VE rTMS $\left(t_{(8)}=\right.$ $3.16, P=0.013)$, whereas the high memory recognition group did not show a significant effect of rTMS on familiarity, $\left(t_{(9)}=\right.$ $1.07, P=0.31$, see Fig. $1 \mathrm{~B}$ ).

Inclusion of the between-subject factor did not considerably alter the main effect of rTMS on recollection $\left(F_{(1,17)}=\right.$ $\left.0.015, P=0.91, \eta_{p}{ }^{2}=0.001\right)$ and familiarity $\left(F_{(1,17)}=0.86, P=0.37, \eta_{p}{ }^{2}=0.048\right)$.

\section{Subjectively perceived confidence}

No main effect of rTMS was found for subjectively perceived confidence in "old" $\left(F_{(1,18)}=2.57, \quad P=0.13, \quad \eta_{p}^{2}=0.13\right)$ and subjectively perceived confidence in "new" responses $\left(F_{(1,18)}=0.69, P=0.42\right.$, $\eta_{p}{ }^{2}=0.37$; see Fig. 2).

The three-way interaction between stimulation (AG, VE), memory measure (subjectively perceived confidence in "old" responses, subjectively perceived confidence in "new" responses), and group (high, low) was significant $\left(F_{(1,17)}=\right.$ $\left.7.59, P=0.014, \eta_{p}{ }^{2}=0.31\right)$. Post-hoc analysis showed that individual differences in memory recognition did not interact with rTMS condition on the subjectively perceived confidence in "new" responses $\left(F_{(1,17)}=2.03, \quad P=0.17, \quad \eta_{p}^{2}=0.11\right)$. In 

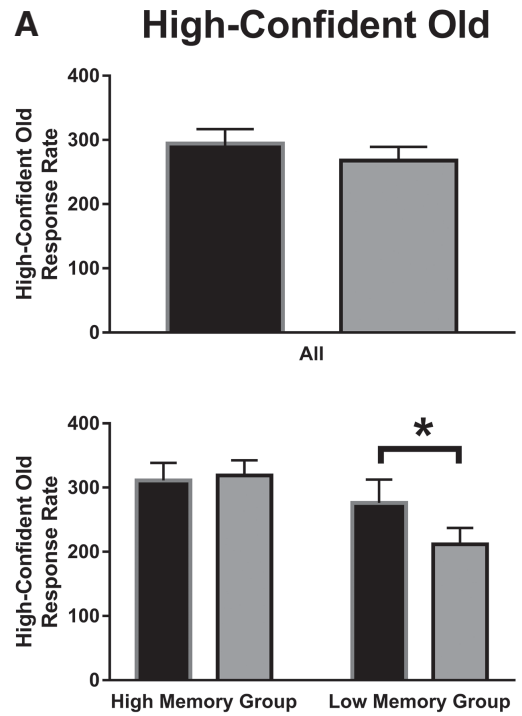

\section{Angular Gyrus}

Figure 2. (Top) Mean high-confident rates for "old" and "new" responses per stimulation condition (Bottom) Mean high-confident rates for "old" and "new" responses for low and high performing participants, based on a d-prime median split. Error bars represent standard errors of the mean. A statistical significant difference $(P<0.05)$ is indicated by a star.

contrast, a significant interaction effect between rTMS condition and individual differences on the subjectively perceived confidence in "old" responses was found $\left(F_{(1,17)}=5.94, P=0.026, \eta_{p}{ }^{2}=\right.$ $0.26)$. Post-hoc $t$-tests revealed that in the low memory recognition group subjectively perceived confidence in "old" responses was enhanced after left AG rTMS as compared to VE rTMS $\left(t_{(8)}=2.62, P=\right.$ 0.031 ). The high memory recognition group did not show a significant effect of rTMS on subjectively perceived confidence in "old" responses $\left(t_{(9)}=0.43, P=0.68\right.$, see Fig. $\left.2 \mathrm{~A}\right)$.

Inclusion of the between-subject factor did not considerably change the main effect of rTMS on subjectively perceived confidence in "old" $\left(F_{(1,17)}=3.75, P=0.070, \eta_{p}{ }^{2}=0.18\right)$ and "new" responses $\left(F_{(1,17)}=0.86, P=0.37, \eta_{p}{ }^{2}=0.048\right)$.

\section{Time course of the rTMS effect}

Since rTMS effects have been shown to decay over time (Thut and Pascual-Leone 2010), it is worthwhile to examine whether stimulation-related adjustments in performance change as a function of time. To this end, we split up retrieval in three equally sized phases of 300 trials. A GLM was performed with phase (early, mid, late), measurement (recollection, familiarity, subjectively perceived confidence in "old" responses, subjectively perceived confidence in "new" responses), and stimulation (AG, vertex) as within subject variables. Of main interest was the three-way interaction between phase, measurement, and stimulation, which was not significant $\left(F_{(3.07,55.28)}=0.98, P=0.41, \eta_{p}{ }^{2}=0.052\right)$. This indicates that stimulation-related changes did not vary as a function of time during retrieval.

\section{Discussion}

Our results show that inhibitory rTMS over the left AG, as compared to inhibitory rTMS over the vertex, does not significantly influence recollection and the subjectively perceived confidence in "new" responses. However, we did find that left AG TMS increased familiarity and subjectively perceived confidence in "old" respons- er brain regions, such as the hippocampal formation, that are more involved in binding episodic representations, which influence the richness of retrieved memories (Eichenbaum 2001; Bergmann et al. 2012; St-Laurent et al. 2014, 2016). The hippocampal formation and the AG are both part of a complex neural network associated with memory (Hayama et al. 2012; Thakral et al. 2017b). A close interaction within this memory network could perhaps explain the increased activation in the left AG during recollection (Skinner and Fernandes 2007; Vilberg and Rugg 2008), even though the left AG might not be directly involved in recollection-related processes. Alternatively, recollection-related left AG activity could actually be due to the involvement of the PPC in factors, such as subjectively perceived memory confidence, which are strongly related to episodic memory and hard to dissociate (Schoo et al. 2011).

Even though the AG has been implicated in familiarity (Skinner and Fernandes 2007; Horn et al. 2016), AG activity is typically linked to recollection (Ciaramelli et al. 2008; Vilberg and Rugg 2008; Rugg and King 2017). We speculate that the rTMS effect on familiarity in our study may, at least in part be due to the recruitment of the left AG in subjectively perceived memory confidence. This speculation is based on the similar effect we found for subjectively perceived memory confidence in "old" responses. In addition, the familiarity estimates are generated with the confident ratings of the participants. Future studies are needed to confirm this speculation.

According to dual-process theories, recollection and familiarity are considered to involve independent retrieval processes. While recollection is characterized by a step-wise (Boolean) process that is accompanied by high levels of subjectively perceived memory confidence, familiarity is proposed to have a linear relation with subjectively perceived memory confidence (Mandler 1980; Yonelinas 2002). In contrast, strength theories assume that memory recognition is based on a single dimension of memory strength. In the latter theory, both recollection and familiarity are proposed to lie on a continuum that can be understood in terms of a varying degree of subjectively perceived confidence (Wixted 2007; Mickes et al. 2009). In concordance, it has been shown 
that activation patterns patterns in the parietal cortex are better explained by strength theories (Hayes et al. 2011). Our results concur with the proposed link between the parietal cortex and strength theories.

Both subjectively perceived memory confidence in "old" responses and familiarity were affected by left AG rTMS, but only in participants with relatively low memory recognition. It has been reported before that the effects of noninvasive brain stimulation vary across individuals (Daskalakis et al. 2006; Pena-Gomez et al. 2011; Berryhill and Jones 2012). Here, we speculate that high performing participants may have already reached an optimal level of performance, resulting in a ceiling effect, while in low performing participants there was still room for improvement (Krause and Cohen Kadosh 2014). Not taking into account individual difference in baseline performance may provide a possible explanation for previous studies that did not find group-level effects of left AG TMS on item memory (Yazar et al. 2014; Bonni et al. 2015). Our findings further illustrate the importance of taking individual variability into account in studying the effects of rTMS on behavioral and neurophysiological indices.

Importantly, our rTMS protocol was intended to disrupt left AG function and worsen performance, but instead we found an increase in subjectively perceived confidence and familiarity. The apparent paradox may imply that inhibition of physiological activity does not mean a decrease in functionality per se (Bonni et al. 2015). Indeed, it has been reported before that inhibitory rTMS does not necessarily lead to disruptive effects on behavior (Thut and Pascual-Leone 2010; Caparelli et al. 2012; Strigaro et al. 2016). Also, on the neurophysiological level inhibitory rTMS protocols may cause facilitatory effects that are state-dependent (Ridding and Ziemann 2010).

For example, according to the Bienenstock-Cooper-Munro (BCM) model (Bienenstock et al. 1982), neurons that are in a less excitable state prior to stimulation can be more susceptible to increased cortical excitability, even after inhibitory TMS. In keeping with fMRI studies that consistently show decreased AG activity during encoding (Huijbers et al. 2011; Elman et al. 2013), subsequent inhibitory rTMS might in fact induce cortical facilitation and result in related functional improvement. In further support of this view, a previous study indeed showed that the application of inhibitory rTMS after memory encoding lead to an increased memory performance (Bonni et al. 2015). As previously discussed, the AG is part of a complex memory network that involves a variety of brain regions including the hippocampus (Hayama et al. 2012; Thakral et al. 2017b). In a recent study $1 \mathrm{~Hz}$ rTMS was found to increase functional connectivity between the left inferior PPC and the hippocampus (Eldaief et al. 2011). Increases in functional connectivity are typically interpreted as improved network functioning (Ranganath et al. 2005; Wang et al. 2010), and may hint toward a possible mechanism that can explain the present "paradoxical facilitation" of memory performance (Kapur 1996; Brem et al. 2014).

Several limitations of this study should be mentioned. First, due to the ad hoc nature of our analyses on individual performance differences, these analyses lack high statistical power. Even though the results concur with recent findings demonstrating state-dependent effects of performance on the efficiency of noninvasive brain stimulation (Sarkar et al. 2014; Benwell et al. 2015; Klaus and Schutter 2018), they should be interpreted with caution. Second, the dual-process signal detection model that we adopted is based on the general assumption that recollection is based on a threshold-like process while familiarity is more related to a continuous process (Yonelinas et al. 1996; Yonelinas 2002). As a consequence, our recollection and familiarity estimates do not hold if recollection, similar to familiarity, is conceptualized as a continuous process. Third, we localized the left AG on the basis of MNI coordinates from a meta-analytic neuroimaging study (Vilberg and Rugg 2008) while we used neuronavigation to position and hold the coil over the target site (Yazar et al. 2014, 2017). However, individual differences in brain anatomy as well as the physiological susceptibility of rTMS give rise to variability (Wild et al. 2017) that may have a negative impact on the effect size (Sack et al. 2009).

In conclusion, our study provides evidence that the left AG is involved in familiarity and subjectively perceived memory confidence, while the left AG does not appear to be involved in recollection. Our study further highlights the complex relationship between TMS-related physiological and behavioral changes, and the importance of taking into account individual differences in performance in experimental designs involving rTMS.

\section{Materials and Methods}

\section{Participants}

Twenty healthy adult right-handed volunteers (11 women) with a mean age of $23(M=23.05, S D=3.41)$ participated in this study. All had normal or corrected-to-normal vision, were native Dutch speakers, nonsmokers and free from neurological or psychiatric conditions (self-report). All participants received written and oral information prior to participation, but remained naive about the aim of the study, and all provided written informed consent. Main exclusion criteria were: metal in their cranium; epilepsy or a family history of epilepsy; history of other neurological conditions or psychiatric disease; heart disease; use of psychoactive medication or substances; pregnancy. Stimulation parameters were in agreement with the International Federation of Clinical Neurophysiology safety guidelines (Rossi et al. 2009), and the study was approved by the medical ethics committee of the Radboud University Medical Center, Nijmegen, The Netherlands, and carried out in accordance with the standards set by the Declaration of Helsinki. One participant was excluded from data analyses due to an at chance level performance on the memory task.

\section{Memory task}

Stimuli were presented on a personal computer screen with a 21 -inch monitor. Stimulus presentation and recording of responses were attained using PsychoPy (v1.80; Peirce 2007). The stimulus material consisted of 900 words per session, differing per participant, randomly chosen from a pool of 2038 words. This set was selected based on word frequency (Dutch logarithmic: $M=0.92$ $(S D=0.71$, range $=[0,3.66])$, familiarity $(M=496.48(S D=73.61$, range $=[203,657])$, and concreteness $(M=527.97(S D=82.19$, range $=[204,670])$, from the MRC Psycholinguistic Database (http://websites.psychology.uwa.edu.au/school/MRCDatabase/uwa mrc.htm) and translated to Dutch (Dutch word length: $M=6.44$ $(S D=2.51$, range $=[2,25])$. In this way, participants were only presented with words that were relatively easy to imagine, which facilitates memory processes (Nittono et al. 2002; Fliessbach et al. 2006). Two parallel versions of word lists were generated per participant, one for each session.

\section{Procedure}

Participants received either rTMS over the left AG or vertex (VE) during two sessions in a randomized and counterbalanced order. Sessions were separated by exactly $1 \mathrm{wk}$ and controlled for time of day. During both sessions, resting motor threshold (MT) for the right first dorsal interosseous hand muscle was determined for each participant (Schutter and van Honk 2006). Next, the participant's head was coregistered to a standard brain using the Localite TMS Navigator (Localite $\mathrm{GmbH}$ ) to guide TMS stimulation.

After the MT was determined, participants were seated in front of a computer and instructed on the encoding phase of the memory task. In this phase of the memory task, trials began with 
Encoding Trials (Pleasant or unpleasant?)
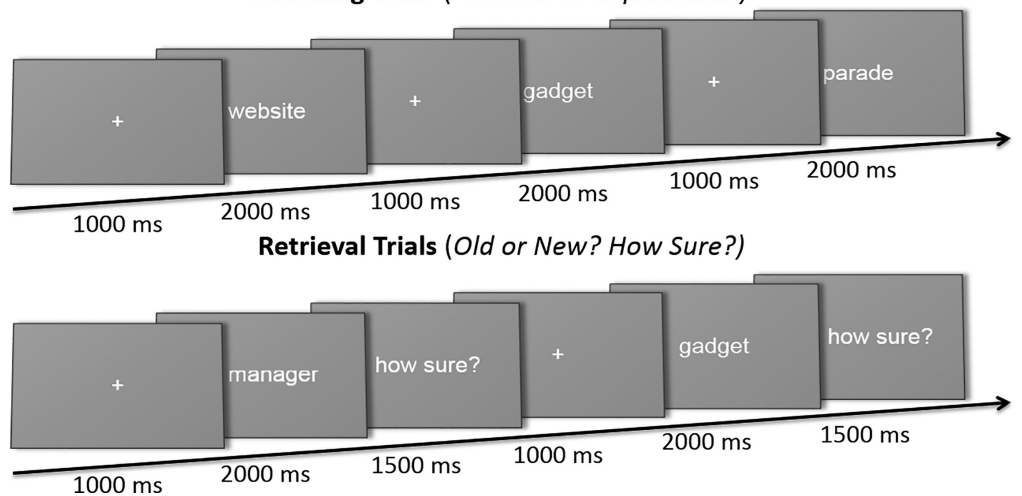

Figure 3. Schematic overview of the memory task. In the encoding phase of the memory task, participants made a semantic classification ("pleasant" or "unpleasant") regarding the presented word. In the retrieval phase, all words shown during encoding were presented again, complemented by new words. Participants indicated whether they saw the word presented in the encoding phase of the memory task ("old" or "new"), and subsequently how sure they were of this classification on the basis of a 3-point scale ("not sure"-_a bit sure"-_definitely sure").

a 1-sec centrally presented fixation cross, followed by a 2-sec presentation of a word. Each participant performed 450 encoding trials, while making a semantic classification ("pleasant" or "unpleasant") regarding the presented word. The sematic classification of the stimuli ensured that participants kept attending to the stimulus presentations and aimed to deepen encoding (see Fig. 3; Demb et al. 1995; Fletcher et al. 2003).

When the encoding phase was completed, the eight-shaped coil (Cool-B65, Magventure A/S) was placed over the target (left AG: (MNI coordinates: $-43,-66,38$; Vilberg and Rugg 2008) or control location (VE: (MNI coordinates: 0, -15, 74; Okamoto et al. 2004; see Figs. 4-5).

After the coil was positioned over the target location, volunteers received a $20 \mathrm{~min} 1 \mathrm{~Hz}$ train of rTMS (1200 pulses) over the left AG or VE at $90 \%$ of MT. The MTs $(M=49.20, S D=9.78)$ did not differ significantly between sessions $\left(t_{(19)}=0.85, P=0.41\right)$ During stimulation, the participant sat quietly in a chair while the TMS coil was held manually by the experimenter. The exact target location of the TMS coil relative to the head was monitored online and adjusted when there was a deviation greater than two millimeter from the target in any of the three dimensions. RTMS was administered intermediate the encoding and retrieval phase of the task in order to interfere with subsequent retrieval processes and to avoid distraction during encoding (Yazar et al. 2014; Bonni et al. 2015).

During the retrieval phase, participants performed a recognition task, including all 450 "old" words presented during encoding and 450 "new" words. In total, each participant performed 900 fixed-paced recognition trials, which started with a 1 sec fixation cross, followed by a 2 sec presentation of a word. During word presentation, participants classified the item as "old" or "new." When a response was made, the trial ended with a "confidence" screen presenting the question "how sure?" for one and a half seconds. During the "confidence" screen participants rated their confidence of their "old/new" classification on a three-point scale (see Fig. 3; Yonelinas et al. 1996).
To familiarize participants with the task, 20 practice trials preceded both the encoding and retrieval phase of the experiment. Stimuli used during the practice trials were not used in the experimental trials. Each trial consisted of a $2 \mathrm{sec}$ word presentation, $1 \mathrm{sec}$ fixation presentation, and one and a half seconds to make confidence judgements. After every 150 experimental trials there was a short break of at least one minute, resulting in $25 \mathrm{~min}$ of encoding and $75 \mathrm{~min}$ of retrieval. At the end of the second session volunteers were debriefed and received compensation for participation.

\section{Data analyses}

Data analyses were performed with the use of MATLAB (v2015b, MathWorks Inc.,). Recollection and familiarity estimates, and d-prime were computed using the ROC toolbox for MATLAB (Koen et al. 2016). Subjectively perceived confidence was measured as the rate of highconfident responses, which was done separately for old trials and new trials.

\section{Statistical analyses}

General linear models (GLMs) for repeated measurements were used to test for significant differences in recollection, familiarity, or confidence, during both stimulation conditions. Previous studies have demonstrated significant inter-individual variability in the behavioral effects of noninvasive brain stimulation (Maeda et al. 2000; Siebner et al. 2009; Pena-Gomez et al. 2011; Klaus and Schutter 2018). To explore such an effect, a median split was made based on d-prime scores obtained during control VE stimulation as a measure of baseline memory recognition. This distinction between sample-based low and high performers was entered as a between-subject factor in the GLMs. Alpha level of significance 


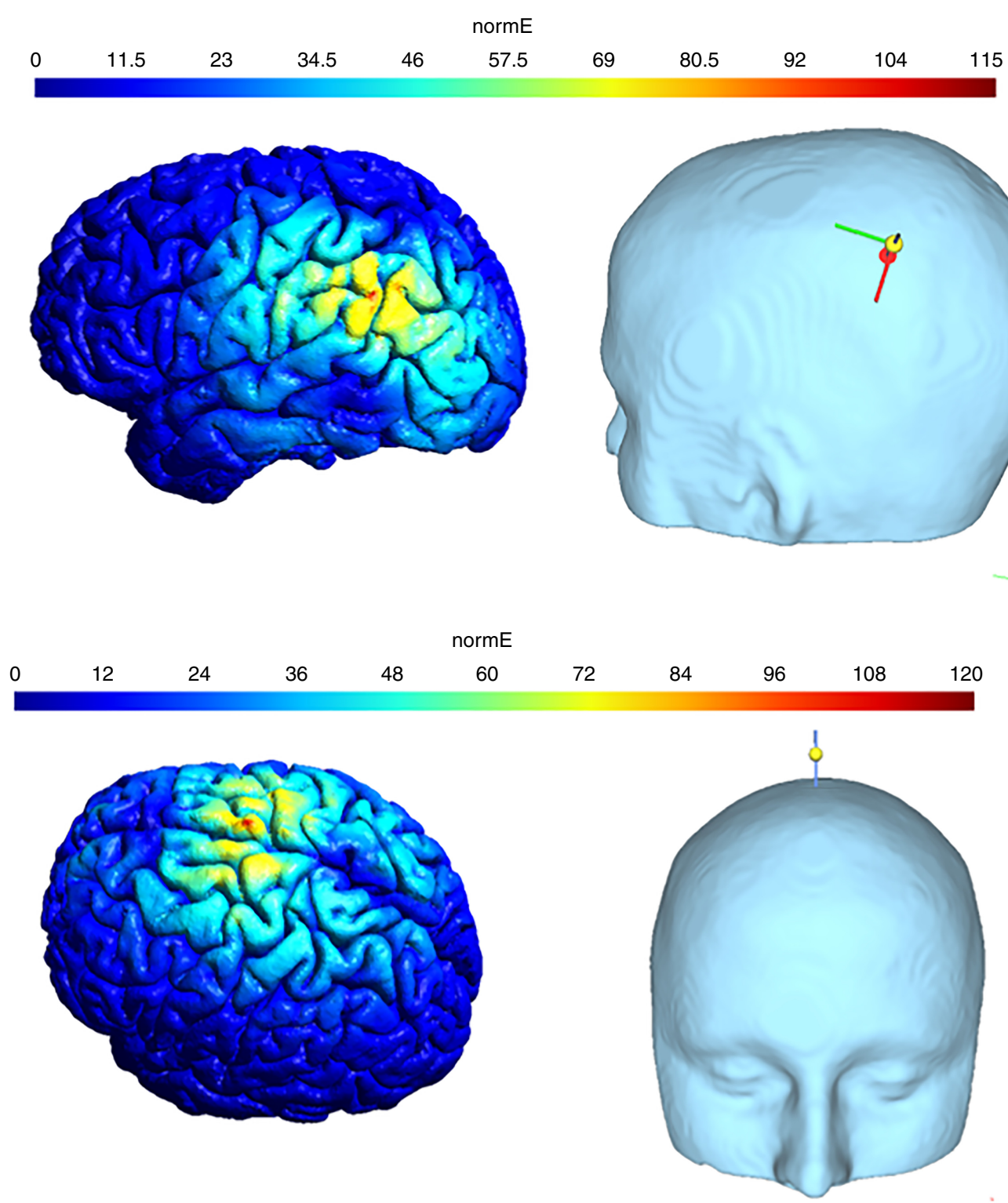

Figure 5. Simulated electric field distribution of stimulation targeting the left AG (top) or VE (bottom), with the use of the SimNIBS software (Opitz et al. 2015). Scalp-cortex distance: $15 \mathrm{~mm}$ (Stokes et al. 2005), changing current strength: $68 \mathrm{~A} / \mathrm{us}$.

was set at 0.05 (two-tailed) and effect sizes (partial eta-squared; $\eta_{p}{ }^{2}$ ) were computed for all analyses.

\section{References}

Ally BA, Simons JS, McKeever JD, Peers PV, Budson AE. 2008. Parietal contributions to recollection: electrophysiological evidence from aging and patients with parietal lesions. Neuropsychologia 46: 1800-1812.

Atkinson RC, Juola JF. 1974. Search and decision processes in recognition memory. WH Freeman, Oxford, England.

Benwell CS, Learmonth G, Miniussi C, Harvey M, Thut G. 2015. Non-linear effects of transcranial direct current stimulation as a function of individual baseline performance: Evidence from biparietal tDCS influence on lateralized attention bias. Cortex 69: 152-165.

Ben-Zvi S, Soroker N, Levy DA. 2015. Parietal lesion effects on cued recall following pair associate learning. Neuropsychologia 73: 176-194.

Bergmann HC, Rijpkema M, Fernández G, Kessels RP. 2012. Distinct neural correlates of associative working memory and long-term memory encoding in the medial temporal lobe. Neuroimage 63: 989-997.
Berryhill ME, Jones KT. 2012. tDCS selectively improves working memory in older adults with more education. Neurosci Lett 521: 148-151.

Berryhill ME, Phuong L, Picasso L, Cabeza R, Olson IR. 2007. Parietal lobe and episodic memory: bilateral damage causes impaired free recall of autobiographical memory. J Neurosci 27: 14415-14423.

Bienenstock EL, Cooper LN, Munro PW. 1982. Theory for the development of neuron selectivity: orientation specificity and binocular interaction in visual cortex. J Neurosci 2: 32-48.

Bonni S, Veniero D, Mastropasqua C, Ponzo V, Caltagirone C, Bozzali M, Koch G. 2015. TMS evidence for a selective role of the precuneus in source memory retrieval. Behav Brain Res 282: 70-75.

Brem AK, Fried PJ, Horvath JC, Robertson EM, Pascual-Leone A. 2014. Is neuroenhancement by noninvasive brain stimulation a net zero-sum proposition? Neuroimage 85(Pt 3): 1058-1068.

Cabeza R, Ciaramelli E, Olson IR, Moscovitch M. 2008. The parietal cortex and episodic memory: an attentional account. Nat Rev Neurosci 9: 613-625.

Caparelli E, Backus W, Telang F, Wang G, Maloney T, Goldstein R, Henn F. 2012. Is $1 \mathrm{~Hz}$ rTMS always inhibitory in healthy individuals? Open Neuroimag J 6: 69-74.

Ciaramelli E, Grady CL, Moscovitch M. 2008. Top-down and bottom-up attention to memory: a hypothesis (AtoM) on the role of the 
posterior parietal cortex in memory retrieval. Neuropsychologia 46: $1828-1851$.

Ciaramelli E, Faggi G, Scarpazza C, Mattioli F, Spaniol J, Ghetti S, Moscovitch M. 2017. Subjective recollection independent from multifeatural context retrieval following damage to the posterior parietal cortex. Cortex 91: 114-125.

Daselaar SM, Fleck MS, Cabeza R. 2006. Triple dissociation in the medial temporal lobes: recollection, familiarity, and novelty. J Neurophysiol 96: 1902-1911.

Daskalakis ZJ, Möller B, Christensen BK, Fitzgerald PB, Gunraj C, Chen R. 2006. The effects of repetitive transcranial magnetic stimulation on cortical inhibition in healthy human subjects. Exp Brain Res 174: 403-412.

Davidson PS, Anaki D, Ciaramelli E, Cohn M, Kim AS, Murphy KJ, Troyer AK, Moscovitch M, Levine B. 2008. Does lateral parietal cortex support episodic memory? Evidence from focal lesion patients. Neuropsychologia 46: 1743-1755.

Demb JB, Desmond JE, Wagner AD, Vaidya CJ, Glover GH, Gabrieli JD. 1995. Semantic encoding and retrieval in the left inferior prefrontal cortex: a functional MRI study of task difficulty and process specificity. J Neurosci 15: 5870-5878.

Eichenbaum H. 2001. The hippocampus and declarative memory: cognitive mechanisms and neural codes. Behav Brain Res 127: 199-207.

Eldaief MC, Halko MA, Buckner RL, Pascual-Leone A. 2011. Transcranial magnetic stimulation modulates the brain's intrinsic activity in a frequency-dependent manner. Proc Natl Acad Sci 108: 21229-21234.

Elman JA, Rosner ZA, Cohn-Sheehy BI, Cerreta AG, Shimamura AP. 2013. Dynamic changes in parietal activation during encoding: implications for human learning and memory. Neurolmage 82: 44-52.

Fletcher PC, Stephenson CM, Carpenter TA, Donovan T, Bullmorel ET. 2003. Regional brain activations predicting subsequent memory success: an event-related fMRI study of the influence of encoding tasks. Cortex 39: 1009-1026.

Fliessbach K, Weis S, Klaver P, Elger CE, Weber B. 2006. The effect of word concreteness on recognition memory. Neuroimage 32: $1413-1421$.

Hayama HR, Vilberg KL, Rugg MD. 2012. Overlap between the neural correlates of cued recall and source memory: evidence for a generic recollection network? J Cogn Neurosci 24: 1127-1137.

Hayes SM, Buchler N, Stokes J, Kragel J, Cabeza R. 2011. Neural correlates of confidence during item recognition and source memory retrieval: evidence for both dual-process and strength memory theories. J Cogn Neurosci 23: 3959-3971.

Horn M, Jardri R, D'Hondt F, Vaiva G, Thomas P, Pins D. 2016. The multiple neural networks of familiarity: a meta-analysis of functional imaging studies. Cogn Affect Behav Neurosci 16: 176-190.

Hower KH, Wixted J, Berryhill ME, Olson IR. 2014. Impaired perception of mnemonic oldness, but not mnemonic newness, after parietal lobe damage. Neuropsychologia 56: 409-417.

Huijbers W, Pennartz CM, Cabeza R, Daselaar SM. 2011. The hippocampus is coupled with the default network during memory retrieval but not during memory encoding. PloS One 6: e17463.

Kapur N. 1996. Paradoxical functional facilitation in brain-behaviour research. A critical review. Brain 119(Pt 5): 1775-1790.

Klaus J, Schutter D. 2018. The role of left dorsolateral prefrontal cortex in language processing. Neuroscience 377: 197-205.

Koen JD, Yonelinas AP. 2014. The effects of healthy aging, amnestic mild cognitive impairment, and Alzheimer's disease on recollection and familiarity: a meta-analytic review. Neuropsychol Rev 24: $332-354$

Koen JD, Barrett FS, Harlow IM, Yonelinas AP. 2016. The ROC Toolbox: a toolbox for analyzing receiver-operating characteristics derived from confidence ratings. Behav Res Methods 49: 1399-1406.

Krause B, Cohen Kadosh R. 2014. Not all brains are created equal: the relevance of individual differences in responsiveness to transcranial electrical stimulation. Front Syst Neurosci 8: 25.

Maeda F, Keenan JP, Tormos JM, Topka H, Pascual-Leone A. 2000. Interindividual variability of the modulatory effects of repetitive transcranial magnetic stimulation on cortical excitability. Exp Brain Res 133: $425-430$.

Mandler G. 1980. Recognizing: the judgment of previous occurrence. Psychol Rev 87: 252.

Mickes L, Wais PE, Wixted JT. 2009. Recollection is a continuous process: implications for dual-process theories of recognition memory. Psychol Sci 20: 509-515.

Moscovitch M, Cabeza R, Winocur G, Nadel L. 2016. Episodic memory and beyond: the hippocampus and neocortex in transformation. Annu Rev Psychol 67: 105-134.

Nilakantan AS, Bridge DJ, Gagnon EP, VanHaerents SA, Voss JL. 2017. Stimulation of the posterior cortical-hippocampal network enhances precision of memory recollection. Curr Biol 27: 465-470.
Nittono H, Suehiro M, Hori T. 2002. Word imageability and N400 in an incidental memory paradigm. Int J Psychophysiol 44: 219-229.

Okamoto M, Dan H, Sakamoto K, Takeo K, Shimizu K, Kohno S, Oda I, Isobe S, Suzuki T, Kohyama K, et al. 2004. Three-dimensional probabilistic anatomical cranio-cerebral correlation via the international 10-20 system oriented for transcranial functional brain mapping. Neuroimage 21: 99-111.

Opitz A, Paulus W, Will S, Antunes A, Thielscher A. 2015. Determinants of the electric field during transcranial direct current stimulation. Neuroimage 109: 140-150.

Peirce JW. 2007. PsychoPy-Psychophysics software in Python. J Neurosci Methods 162: 8-13.

Pena-Gomez C, Vidal-Pineiro D, Clemente IC, Pascual-Leone A, BartresFaz D. 2011. Down-regulation of negative emotional processing by transcranial direct current stimulation: effects of personality characteristics. PLoS One 6: e22812.

Ranganath C, Heller A, Cohen MX, Brozinsky CJ, Rissman J. 2005. Functional connectivity with the hippocampus during successful memory formation. Hippocampus 15: 997-1005.

Ridding MC, Ziemann U. 2010. Determinants of the induction of cortical plasticity by non-invasive brain stimulation in healthy subjects. JPhysiol 588: 2291-2304.

Rossi S, Hallett M, Rossini PM, Pascual-Leone A, Safety of TMS Consensus Group. 2009. Safety, ethical considerations, and application guidelines for the use of transcranial magnetic stimulation in clinical practice and research. Clin Neurophysiol 120: 2008-2039.

Rugg MD, King DR. 2017. Ventral lateral parietal cortex and episodic memory retrieval. Cortex. doi: 10.1016/j.cortex.2017.07.012

Sack AT, Cohen Kadosh R, Schuhmann T, Moerel M, Walsh V, Goebel R. 2009. Optimizing functional accuracy of TMS in cognitive studies: a comparison of methods. J Cognit Neurosci 21: 207-221.

Sarkar A, Dowker A, Cohen Kadosh R. 2014. Cognitive enhancement or cognitive cost: trait-specific outcomes of brain stimulation in the case of mathematics anxiety. J Neurosci 34: 16605-16610.

Schoo LA, van Zandvoort MJ, Biessels GJ, Kappelle LJ, Postma A, de Haan EH. 2011. The posterior parietal paradox: Why do functional magnetic resonance imaging and lesion studies on episodic memory produce conflicting results? J Neuropsychol 5: 15-38.

Schutter DJ, van Honk J. 2006. A standardized motor threshold estimation procedure for transcranial magnetic stimulation research. J ECT 22: 176-178.

Siebner HR, Hartwigsen G, Kassuba T, Rothwell JC. 2009. How does transcranial magnetic stimulation modify neuronal activity in the brain? Implications for studies of cognition. Cortex 45: $1035-1042$.

Simons JS, Peers PV, Hwang DY, Ally BA, Fletcher PC, Budson AE. 2008. Is the parietal lobe necessary for recollection in humans? Neuropsychologia 46: $1185-1191$.

Simons JS, Peers PV, Mazuz YS, Berryhill ME, Olson IR. 2010. Dissociation between memory accuracy and memory confidence following bilateral parietal lesions. Cereb Cortex 20: 479-485.

Skinner EI, Fernandes MA. 2007. Neural correlates of recollection and familiarity: a review of neuroimaging and patient data. Neuropsychologia 45: 2163-2179.

St-Laurent M, Moscovitch M, Jadd R, McAndrews MP. 2014. The perceptual richness of complex memory episodes is compromised by medial temporal lobe damage. Hippocampus 24: 560-576.

St-Laurent M, Moscovitch M, McAndrews MP. 2016. The retrieval of perceptual memory details depends on right hippocampal integrity and activation. Cortex 84: 15-33.

Stokes MG, Chambers CD, Gould IC, Henderson TR, Janko NE, Allen NB, Mattingley JB. 2005. Simple metric for scaling motor threshold based on scalp-cortex distance: application to studies using transcranial magnetic stimulation. J Neurophysiol 94: 4520-4527.

Strigaro G, Hamada M, Cantello R, Rothwell J. 2016. Variability in response to $1 \mathrm{~Hz}$ repetitive TMS. Clin Neurophysiol 127: e40.

Thakral PP, Madore KP, Schacter DL. 2017a. A role for the left angular gyrus in episodic simulation and memory. J Neurosci 37: 8142-8149.

Thakral PP, Wang TH, Rugg MD. 2017b. Decoding the content of recollection within the core recollection network and beyond. Cortex 91: 101-113.

Thut G, Pascual-Leone A. 2010. A review of combined TMS-EEG studies to characterize lasting effects of repetitive TMS and assess their usefulness in cognitive and clinical neuroscience. Brain Topogr 22: $219-232$.

Vilberg KL, Rugg MD. 2008. Memory retrieval and the parietal cortex: a review of evidence from a dual-process perspective. Neuropsychologia 46: 1787-1799.

Wang L, Negreira A, LaViolette P, Bakkour A, Sperling RA, Dickerson BC. 2010. Intrinsic interhemispheric hippocampal functional connectivity predicts individual differences in memory performance ability. Hippocampus 20: 345-351. 
Wang JX, Rogers LM, Gross EZ, Ryals AJ, Dokucu ME, Brandstatt KL, Hermiller MS, Voss JL. 2014. Targeted enhancement of corticalhippocampal brain networks and associative memory. Science 345: $1054-1057$.

Wild HM, Heckemann RA, Studholme C, Hammers A. 2017. Gyri of the human parietal lobe: volumes, spatial extents, automatic labelling, and probabilistic atlases. PLoS One 12: e0180866.

Wixted JT. 2007. Dual-process theory and signal-detection theory of recognition memory. Psychol Rev 114: 152-176.

Woodruff CC, Hayama HR, Rugg MD. 2006. Electrophysiological dissociation of the neural correlates of recollection and familiarity. Brain Res 1100: 125-135.

Yazar Y, Bergstrom ZM, Simons JS. 2014. Continuous theta burst stimulation of angular gyrus reduces subjective recollection. PLoS One 9: e110414.

Yazar Y, Bergstrom ZM, Simons JS. 2017. Reduced multimodal integration of memory features following continuous theta burst stimulation of angular gyrus. Brain stimulation 10: 624-629.

Yonelinas AP. 2001a. Components of episodic memory: the contribution of recollection and familiarity. Philos Trans R Soc Lond B Biol Sci 356: 1363-1374.
Yonelinas AP. 2001b. Consciousness, control, and confidence: the 3 Cs of recognition memory. J Exp Psychol Gen 130: 361-379.

Yonelinas AP. 2002. The nature of recollection and familiarity: a review of 30 years of research. J Mem Lang 46: 441-517.

Yonelinas AP, Jacoby LL. 1995. Dissociating automatic and controlled processes in a memory-search task: beyond implicit memory. Psychol Res 57: $156-165$.

Yonelinas AP, Levy BJ. 2002. Dissociating familiarity from recollection in human recognition memory: different rates of forgetting over short retention intervals. Psychon Bull Rev 9: 575-582.

Yonelinas AP, Dobbins I, Szymanski MD, Dhaliwal HS, King L. 1996. Signal-detection, threshold, and dual-process models of recognition memory: ROCs and conscious recollection. Conscious Cogn 5: 418-441.

Yonelinas AP, Otten LJ, Shaw KN, Rugg MD. 2005. Separating the brain regions involved in recollection and familiarity in recognition memory. J Neurosci 25: 3002-3008.

Received June 12, 2018; accepted in revised form June 15, 2018. 


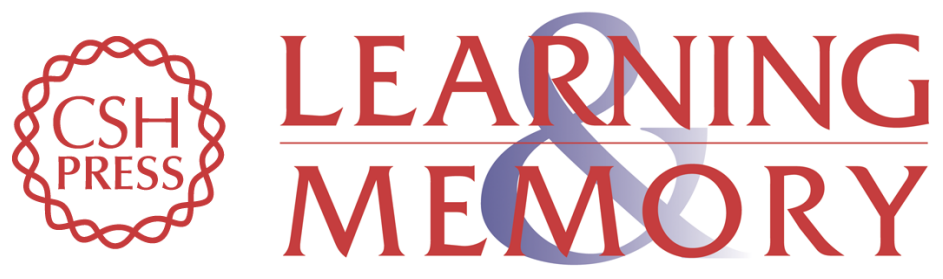

\section{The posterior parietal cortex and subjectively perceived confidence during memory retrieval}

Syanah C. Wynn, Marc P. H. Hendriks, Sander M. Daselaar, et al.

Learn. Mem. 2018, 25:

Access the most recent version at doi:10.1101/Im.048033.118

References This article cites 76 articles, 8 of which can be accessed free at:

http://learnmem.cshlp.org/content/25/8/382.full.html\#ref-list-1

Creative This article is distributed exclusively by Cold Spring Harbor Laboratory Press for the

Commons

first 12 months after the full-issue publication date (see

License

http://learnmem.cshlp.org/site/misc/terms.xhtml). After 12 months, it is available under a Creative Commons License (Attribution-NonCommercial 4.0 International), as described at http://creativecommons.org/licenses/by-nc/4.0/.

Email Alerting Receive free email alerts when new articles cite this article - sign up in the box at the Service top right corner of the article or click here. 